\title{
Key Characteristics of Carbonized Corncob through Hydrothermal and Pyrolysis Conversion Techniques for Further Activation
}

\author{
Nongnoot Srilek, Pruk Aggarangsi
}

\begin{abstract}
The eco-friendly technique which converted the low cost waste residues such as corncob to the valuable material such as solid fuel or advanced material is favorable. This study aims to enhance the knowledge limitation of key elements characteristics of carbonized corncob including fiber constituents, Brunauer-Emmett-Teller surface area and Fourier Transform Infrared spectroscopy as the precursor material for further activation to produce the bio-based activated carbon via the mild temperature hydrothermal technique with demonstrated scale reactor comparing to high temperature pyrolysis. The hydrothermal carbonization takes place in 10 liters reactor at 250 ${ }^{\circ} C$. The pyrolysis is operated at 480 o $C$. The Brunauer-Emmett-Teller surface area of corncob feedstock, hydrochar derived from hydrothermal and biochar derived from pyrolysis are 16.13, 11.53 and $7.66 \mathrm{~m}^{2} \mathrm{~g}^{-1}$ respectively. The oxygenated functional groups contents and high BET surface area of hydrochar are more predominant than biochar. Henceforth, the optimization for better degradation of fiber constituents will be the future work before the activation step.
\end{abstract}

Keywords: Bio-based activated carbon, Hydrochar, Hydrothermal carbonization, Pyrolysis

\section{INTRODUCTION}

The smog or haze crisis in the Northern Thailand is the one major concerns. The open burning of agricultural wastes, especially corn residues, is known to be one of the main causes. The conversion of low value residue biomass interests and challenges researchers in many ways. In recent year, the biomass conversion technology is the key role among many technologies to push the renewable energy stream. The scenario of biomass conversion pathways are more obvious than the former. An alternative and renewable method to efficiently manage the corn residues via biomass conversion is interesting and possible. Corn residues including cobs, leaves and stalks are lignocellulosic materials. Lignocellulosic materials are the biomass mainly composed of cellulose, hemicellulose and lignin. These fiber contents vary from biomass to biomass. To overcome the obstacle of converting the residue biomass which is classified as complex structure to high value material, the chemistry knowledge of materials by means of physicochemical characterization

Revised Version Manuscript Received on April 19, 2019.

Nongnoot Srilek, Department of Mechanical Engineering, Faculty of Engineering, Chiang Mai University, 239 Huay Kaew Road, Muang District, Chiang Mai Thailand

Pruk Aggarangsi , Department of Mechanical Engineering, Faculty of Engineering, Chiang Mai University, 239 Huay Kaew Road, Muang District, Chiang Mai Thailand

aspects co-work with engineering knowledge are performed. Both biomass conversion technologies namely pyrolysis and hydrothermal carbonization are thermochemical conversion technology that convert biomass to the valuable materials for many applications such as fuel, adsorbent, high value chemical. Adsorption from low cost biomass is the expected application.

Cellulose is a linear homo polymer composed of glucose molecule (C6H12O6). At room temperature, cellulose is insoluble in water, dilute acid solution and alkaline solution. General chemical formula of cellulose is represented as (C6H10O5)n. Hemicellulose, (C5H10O5)n, is copolymer with varieties of monomers which differ from cellulose (homo polymer with glucose monomer ). The varieties of monomers resulted in different composition and structure for each biomass. Hemicellulose has lowered the degree of polymerization than cellulose and small crystalline structure. Therefore, hemicellulose is more easily decomposed than cellulose. Lignin is the complex polyphenolic polymer with a three-dimension network which has aromatic monomer included many hydroxyl and polar functional groups. Lignin is insoluble in any solvent [1]. The elemental compositions of cellulose are $43.2 \%$ carbon, $5.7 \%$ hydrogen and $51.1 \%$ oxygen and lignin are $66.0 \%$ carbon, $6.0 \%$ hydrogen and $26.9 \%$ oxygen [2]. Hemicellulose, cellulose and lignin constituents in lignocellulosic materials are decomposed at 180-290, 240-350 and 280-500 o C respectively [3].

The pyrolysis is thermochemical biomass conversion method categorized into slow, fast and flash pyrolysis depend on the residence time and heating rate as Table 1. The temperature ranges for pyrolysis experimental depends on the desired the pyrolytic products. The products from pyrolysis are often called biochar. Biochar is defined as the solid material obtained from thermochemical conversion of biomass in an oxygen-limited environment. Hydrogen/carbon $(\mathrm{H} / \mathrm{C})$ molar ratio of biochar relate to the degree of charring. The 0.7 maximum $\mathrm{H} / \mathrm{C}$ and $10 \%$ carbon are fundamental requirement [4].

The hydrothermal technology is classified into 3 processes as hydrothermal carbonization (HTC), hydrothermal liquefaction (HTL) and hydrothermal gasification (HTG) as Figure 1. The Hydrothermal carbonization (HTC) is the biomass conversion to solid material enriched with carbon. 
Both of low and high moisture content biomass can be converted by HTC especially wet biomass. Dispersion of biomass in water medium is heated up until it reaches the mild

reaction temperature between $180-250$ o C.

Table 1. Temperature, residence time and heating rate of pyrolysis and hydrothermal process

\begin{tabular}{lcccc}
\hline Thermochemical process & $\begin{array}{c}\text { Temperature } \\
\left({ }^{\circ} \mathrm{C}\right)\end{array}$ & $\begin{array}{c}\text { Heating rate } \\
\left({ }^{\circ} \mathrm{C} / \mathrm{min}\right)\end{array}$ & Residence time & References \\
\hline Slow pyrolysis & $400-600$ & $10-30{ }^{\circ} \mathrm{C} / \mathrm{min}$ & $\mathrm{min}-$ days & {$[5],[6]$} \\
Fast pyrolysis & $400-600$ & $>100{ }^{\circ} \mathrm{C} / \mathrm{min}$ & about $1 \mathrm{sec}$ & {$[5],[6]$} \\
Hydrothermal carbonization & $180-250$ & $5-10{ }^{\circ} \mathrm{C} / \mathrm{min}$ & $1-12 \mathrm{~h}$ & {$[5],[7]$} \\
\hline
\end{tabular}

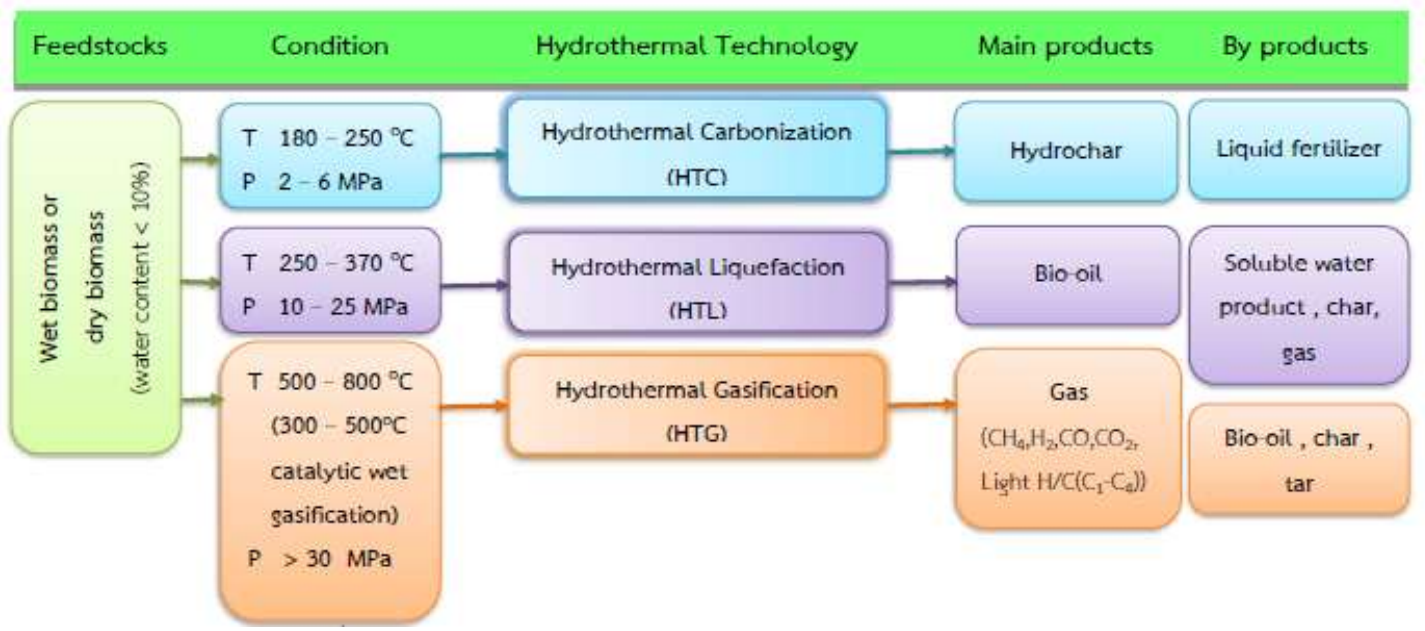

Figure 1. Hydrothermal technology classification

The HTC reaction proceed in pressurized vessel reactor between 2-6 MPa (20-60 bar) [6] which the pressure selfgenerated by heating the reactor. The water medium at these condition, the reaction temperature 180-250 o $\mathrm{C}$ and pressure 2-6 MPa (20-60 bar) which water still maintain in liquid form, is the sub critical water [8]. The favorable product from hydrothermal carbonization is hydrochar or HTC char specifically refers to char produced under hot compressed water medium. The word "hydrochar" emphasizes the disparity of char production process. Hydrochar is defined as the product of hydrothermal carbonization or liquefaction. It is distinct from biochar due to its production process and properties. Moreover, it typically has high $\mathrm{H} / \mathrm{C}$ ratio and lower aromaticity than biochar as well as little or no fused aromatic ring structures [4]. Hydrothermal treatment creates much more oxygen-containing groups on the char's surface [9]. Therefore, typical oxygen/carbon (O/C) and hydrogen/carbon $(\mathrm{H} / \mathrm{C})$ molar ratio of HTC char are higher than pyrolytic char.

The corncob residues, the hemicellulose-depleted waste from furfural production process, were upgraded via HTC for high energy recovery purpose. The high heating value increase $47 \%$ after treatment with an optimum temperature at $230 \mathrm{o} \mathrm{C}$ and 1.5 hour [10]. The prolonged residence time effected on the increase of high heating value beyond 0.5 hour at 250 o C of 0.5 to 6 hour [11]. Arellano et al. [12] reported the energy densification of corncob hydrochar increased with reaction temperature at $180,200,250$ and $350 \mathrm{o} \mathrm{C}$. The most of characterization of hydrochar including thermogravimetric analysis (TGA), Fourier Transform Infrared (FT-IR) Spectroscopy and scanning electron microscopy (SEM) verified the properties at different conditions focusing on an energy performance purpose except the specific adsorption of
Blue Methylene. However, the decrease of specific adsorption with higher hydrothermal temperature could not be conclusive in this study. Nakason et al. [13] reported the optimal condition production of hydrochar from corncob as solid fuel which was determined at $200 \mathrm{oC}, 3$ hour of residence time and biomass to water ratio of 1:5 with maximum energy yield of $68.74 \%$. Magdeldin et al. [14] presented the techno-economic performance indicators of integrated hydrothermal liquefaction which densify forest residue lignocellulosic into liquid fuels and combined heat and power production. However, the many former researches in HTC extend the possibility of corncob feedstock as energy utilization.

Activated carbon is defined as a pyrogenic carbonaceous material (PCM) that has undergone activation, for example by using steam or additions of chemicals [4]. Also, it includes wide range of amorphous carbonaceous materials that exhibit a high degree of porosity and extended inter particulate surface area [15]. The commonly used feedstocks for traditional activated carbon production are wood, coal, petroleum residues, peat, lignite and polymers, which are very expensive and non-renewable [16]. Therefore, the bio-based activated carbon is the expected renewable material. The exertion of bio-sorbent utilization from simple to complex method were studied. Chen et al. [17] investigated the influence of different impregnation ratios, activation temperatures and times on the production of activated carbon using hydrochar produced from condition at $250 \mathrm{o} C$ reaction 
temperature and 4 hour of residence time [11] which the results showed the specific surface area and total pore volume from $5.69 \mathrm{~m} 2 \mathrm{~g}-1$ and $0.136 \mathrm{~cm} 3 \mathrm{~g}-1$ to $2195 \mathrm{~m} 2 \mathrm{~g}-1$ and 1.269 $\mathrm{cm} 3 \mathrm{~g}-1$ under temperature at 400 o $\mathrm{C}$ temperature, 1 hour of residence time and impregnation ratio of 3 .

The char characterization will specify the suitable application such as solid fuel, precursor for making for catalyst, soil amendment, adsorbent etc. Moreover, the in-depth characterization clarifies and enhances the correct upgrading requirement. Due to the many former researches in HTC extend the possibility of corncob feedstock as energy utilization. Therefore, this study aims to understand the important properties of carbonized corncob as carbon precursor derived from biomass. The significant char properties of both high temperature pyrolysis and mild temperature hydrothermal which have been analyzed include fiber contents (by Detergent method), Brunauer-Emmett-Teller (BET) surface area and Fourier Transform Infrared (FT-IR) spectroscopy. The sufficient knowledge will support the process selection and optimized factors which focus on properties including fiber contents after HTC treatment, specific surface area and oxygenated functional groups contents for further activation on the future work. In consequence, this study aims to characterize carbonized corncob through pyrolysis and hydrothermal conversion techniques as precursor for further activation in the adsorption application.

\section{MATERIALS AND METHOD}

\section{A. Materials}

Corncob, an enormous amount agricultural waste residues in Northern Thailand and one of the main causes of smog or haze crisis from open burning, is the selected feedstock in this research. The corncob feedstock as shown in Figure 2 was shortened and sieved with size 10-20 mesh (2-0.841 mm.). The sieved corncob sample is dispersed in deionized water for hydrothermal conversion.

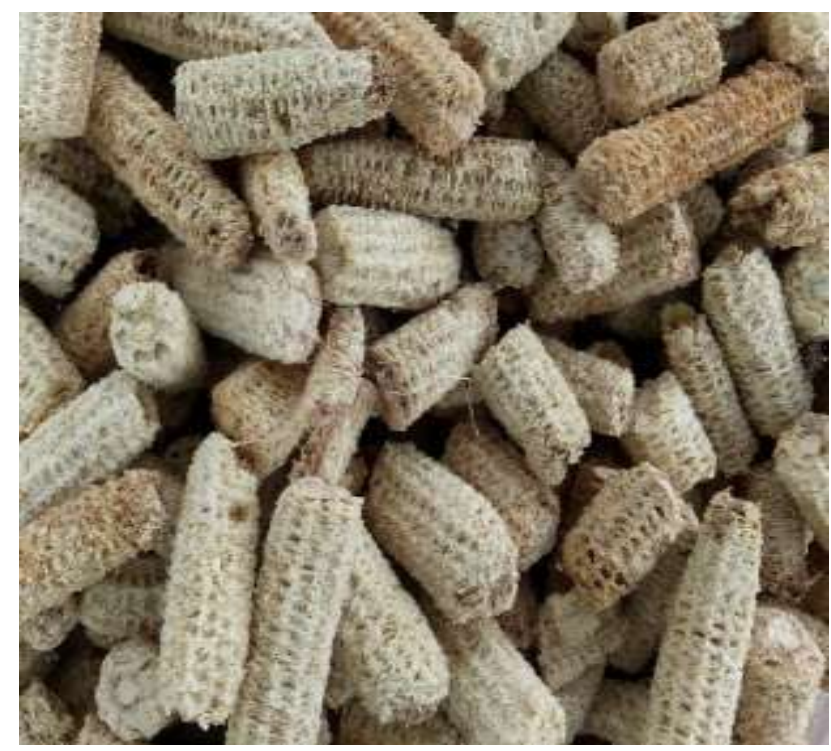

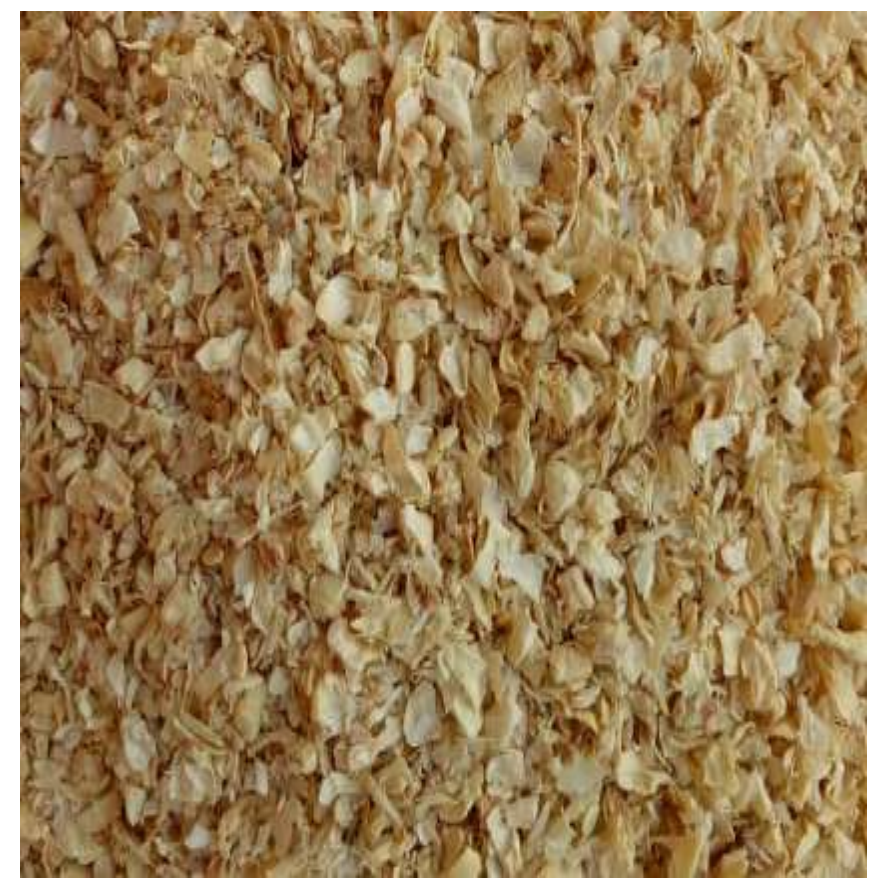

Figure 2. Corncob feedstock

\section{B. Experimental Methods}

Pyrolytic char or biochar is produced from the slow pyrolysis reactors. Laboratory scale pyrolysis reactor is performed in $10 \mathrm{o}$ C/minute and 15 minute of residence time. The temperature at intersection of the thermogravimetric analysis (TGA) plot and derivative plot (DTGA) of corn stover was 420-470 o $\mathrm{C}$ depending on the heating rate [18]. In consequence, the reaction temperature of this study is operated at 480 o $\mathrm{C}$. The flow rate of $\mathrm{N} 2$ for pyrolysis is 1.5 1/minute (Supported by Thermal System Research Unit, Chiang Mai University).The suspension of sieved corncob in deionized water medium filled in 10 liters pressurized vessel reactor (Supported by Energy Research and Development Institute, Chiang Mai University as shown in Figure 3). HTC char or hydrochar is produced from hydrothermal reactor. The hydrothermal carbonization process takes place in deionized water medium with reaction temperature at $250 \mathrm{o} \mathrm{C}$ with maximum specific surface area $\left(8.033 \mathrm{~m}^{2} \mathrm{~g}-1\right)$ while higher temperature tends to reduce specific surface area [8]. The residence time and heating rate of hydrothermal carbonization process are 100 minutes and $5 \mathrm{o} \mathrm{C} /$ minute. The corncob to deionized water (CAS No. 7732-18-5, RCI Labscan) ratio is $1: 20 \mathrm{w} / \mathrm{w}$ for the hydrothermal production. Hydrochar from HTC process was rinsed by deionized water several times and dry at $10{ }^{\circ} \mathrm{C}$ with 12 hour. Both chars are kept in sealable bag for characterization.

\section{Characterization Methods}

Fiber analysis by detergent method, Fourier Transform Infrared (FT-IR) spectroscopy with attenuated total reflection (ATR) technique, and Brunauer-Emmett-Teller (BET) surface area or BET surface area are used for char characterization. Fiber analysis by detergent method analyses the NDF (Neutral Detergent Fibers), ADF (Acid Detergent 
International Conference on Recents Advancements in Engineering and Technology (ICRAET-18) |15th and 16th March 2019|Siddhartha Institute of Technology \& Sciences, Telangana, India

Fibers), and ADL (Acid Detergent Lignin). NDF indicates cellulose, hemicellulose and lignin content. ADF indicates cellulose and lignin content. ADL defines the lignin content. FT-IR spectroscopy is the qualitative analysis for estimation oxygenated functional groups (OFG) contents. The BET surface area is analyzed by Quantachrome surface area and pore size analyzer. BET method use $\mathrm{N} 2$ gas as adsorbate, operating temperature at $150 \mathrm{o} \mathrm{C}$ and relative vapor pressure at $0.05-0.35$.

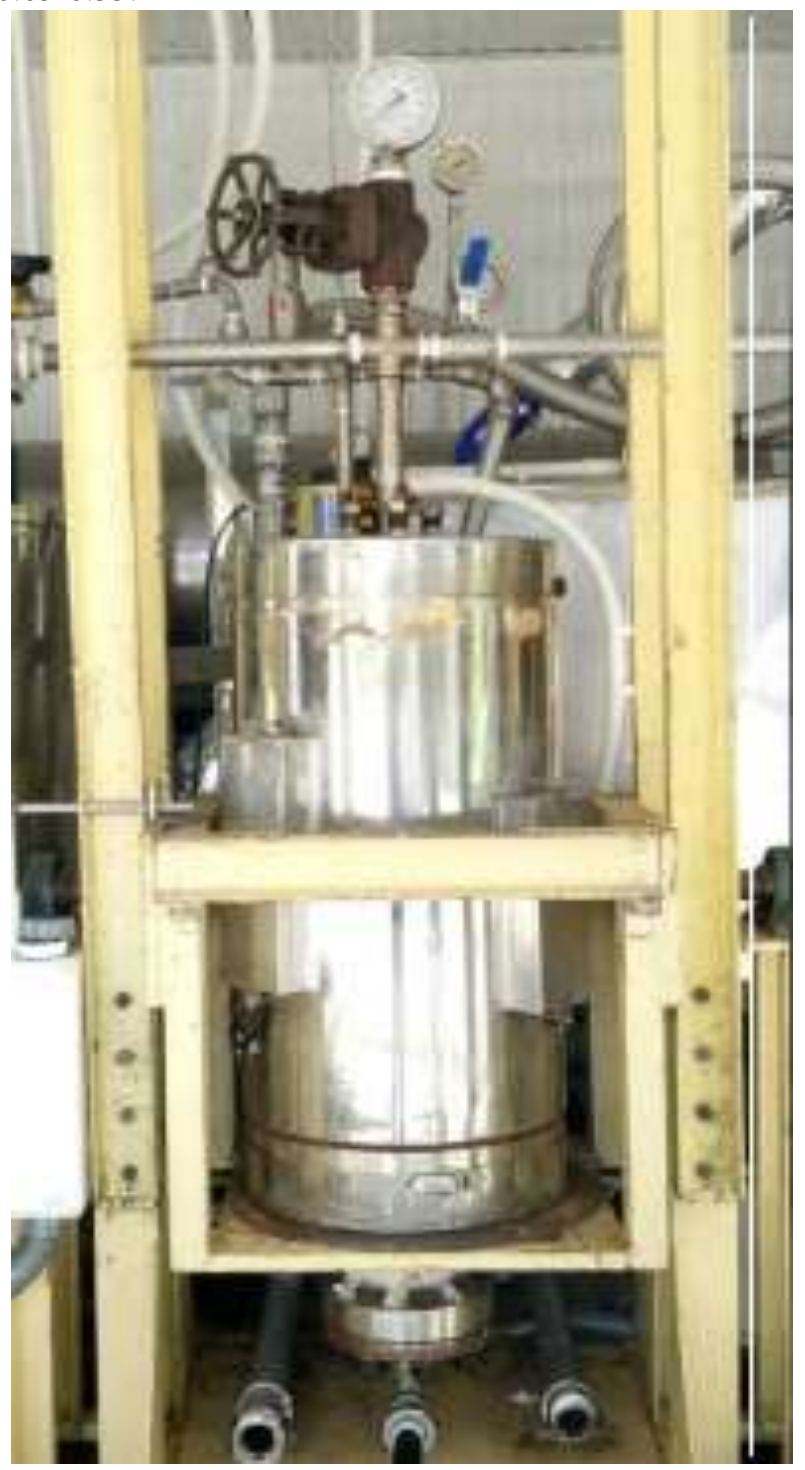

Figure 3. Hydrothermal carbonization reactor (HTC reactor)

(Energy Research and Development Institute, Chiang Mai University)

\section{RESULTS AND DISCUSSION}

In this study, the 3 samples are abbreviated as "CC" (Corncob feedstock with 10-20 mesh size), "B-480" (biochar produced from the laboratory pyrolysis reactor) and "H-250" (hydrochar produced from HTC reactor). The carbonized corncob is demonstrated in the Figure 4.
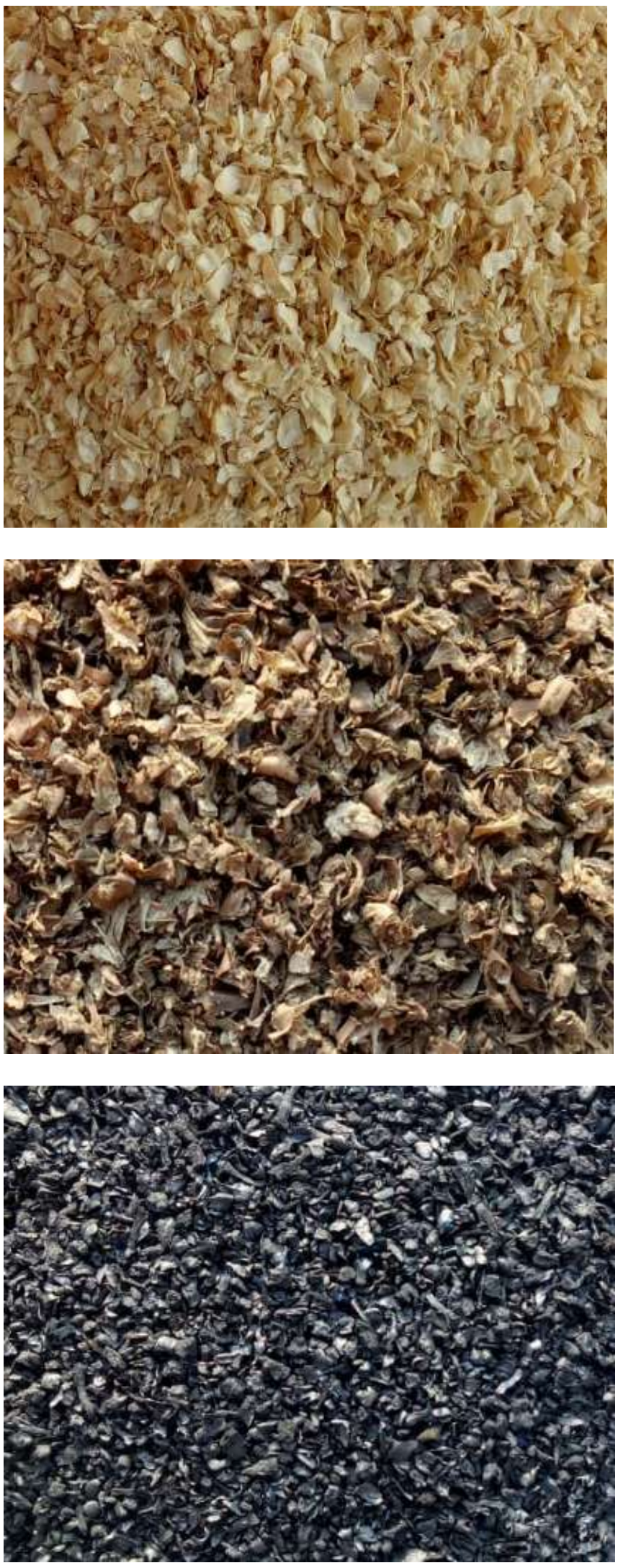

Figure 4. (a) Corncob feedstock with 10-20 mesh size, CC

(b) Hydrochar from Hydrothermal process

at $250 \mathrm{o} \mathrm{C}, \mathrm{H}-250$

(c) Biochar from Pyrolysis process

at 480 o C , B-480

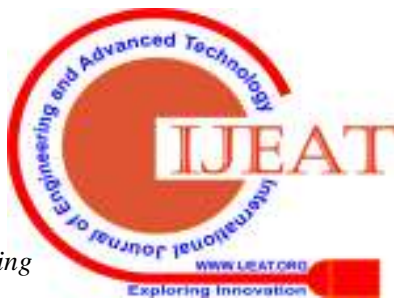




\section{A. Fiber Analysis}

Fiber composition of lignocellulosic material determined by detergent method results in percentage of Neutral Detergent Fibers (NDF), Acid Detergent Fibers (ADF) and Acid Detergent Lignin (ADL). NDF indicate cellulose, hemicellulose and lignin content. ADF value defines the cellulose and lignin. ADL indicates lignin content. NDF, ADF and ADL are interpreted in percentage of cellulose, hemicellulose and lignin which generally understanding as demonstrated in Table 2.

Fiber contents of corncob are $37.15 \%$ cellulose, $40.73 \%$ hemicellulose and $7.22 \%$ lignin. This result shows that hemicellulose is the largest portions and is close to cellulose content. Biochar from pyrolysis conversion, B-480, has $89.36 \%$ lignin. B-480 shows the absolute decomposition of cellulose. The rest hemicellulose from pyrolysis process is less which could be neglected. Lignin content of hydrochar, $\mathrm{H}-250$, produced through the mild condition hydrothermal process at $250 \mathrm{o} \mathrm{C}$ and 100 minutes of residence time slightly increase comparing to feedstock.

The significant factors of HTC including reaction temperature, residence time, heating rate, biomass concentration and catalytic aspects should be taken into account. However, this study focuses on the adsorption application which the surface properties of both char will be discussed in the next section. The fiber analysis and process conditions of feedstock and sample are concluded in Table 3.

Under hydrothermal conditions, cellulose hydrolyzed significantly above 200 o C [19], hemicellulose degraded around 180 o C and lignin decomposed at 280 o C [20]. As the references data of degradation temperature of 3 constituents, HTC char requires the adaptation and optimization. For pyrolysis process as shown in Table 2, B-480, whole cellulose decompose, small amount of hemicellulose are detected and lignin content increase corresponding to the fully carbonized at high reaction temperature. In case of hydrothermal with reaction temperature at 250 o $\mathrm{C}$, hemicellulose content decrease compare to corncob feedstock while increasing of lignin.

As present knowledge, hydrochar formation occur via two pathways - solid-solid conversion and aqueous-solid conversion have reaction sequence including hydrolysis, dehydration, polymerization and condensation. A lignin content resulted in the initial structure of the raw lignocellulose being retained in the solid product while only soluble constituents being converted [15]. However, mechanism of hydrochar formation is still ambiguous. As for the two formation pathways under hydrothermal process, all fiber components can be converted to char but not fully carbonized in the mild condition differ from the solid-solid high pyrolysis temperature.

\section{B. Brunauer-Emmett-Teller surface area (BET surface area)}

BET surface area plays a significant role in studies of adsorption because it represents the shapes of the actual isotherm. And BET equation isotherm is the most important analysis for physical adsorption of gases and vapors on porous carbon [21]. The carbonized Palm Empty fruit bunches produces BET surface area of 6.079, 8.033 and 2.043 $\mathrm{m} 2 \mathrm{~g}-1$ with reaction temperature at 150,250 and 350 o C respectively while palm empty fruit bunches feedstock exhibits $1.478 \mathrm{~m} 2 \mathrm{~g}-1$ BET surface area [8]. The cellulose hydrochar's BET surface area decreases from $27-8 \mathrm{~m} 2 \mathrm{~g}-1$ with the increase of reaction temperature from 230-270 o C [22]. In this study, the BET surface area of char from pyrolysis and hydrothermal with $7.66 \mathrm{~m} 2 \mathrm{~g}-1$ and $11.53 \mathrm{~m} 2$ g-1 shown in Table 4. The BET surface area of corn stover's char derived pyrolysis process (at $550 \mathrm{o} \mathrm{C}, 15$ minutes) and hydrothermal process (at $250 \mathrm{o} \mathrm{C}, 4$ hour) are 12 and $4 \mathrm{~m} 2 \mathrm{~g}-1$ respectively [23] which is found in different trend from this study.

BET C-constant is the one of the two most significant parameters from BET equation including BET surface area. The relationship of BET surface area and BET C-constant is contrary but regular linearity corresponding with increasing reaction temperature demonstrated in Table 4. Char from pyrolysis, B-480, has the lowest BET surface with highest BET C-constant.

Table 2. Fibers analysis by Detergent method and fiber composition

\begin{tabular}{|c|c|c|c|c|c|c|}
\hline \multirow{2}{*}{$\begin{array}{l}\text { Tested } \\
\text { sample }\end{array}$} & \multicolumn{3}{|c|}{$\%$} & \multicolumn{3}{|c|}{$\%$} \\
\hline & $\mathrm{NDF}^{1}$ & $\mathrm{ADF}^{2}$ & $\mathrm{ADL}^{3}$ & Cellulose & Hemicellulose & Lignin \\
\hline $\mathrm{CC}$ & 85.09 & 44.30 & 7.22 & 37.15 & 40.73 & 7.22 \\
\hline B-480 & 77.65 & 74.95 & 89.36 & N/A & 2.69 & 89.36 \\
\hline $\mathrm{H}-250$ & 91.91 & 56.38 & 10.26 & 46.12 & 35.23 & 10.26 \\
\hline
\end{tabular}


$1 \mathrm{NDF}=$ Neutral Detergent Fibers $\quad($ cellulose,$\quad 3 \mathrm{ADL}=$ Acid Detergent Lignin (lignin) hemicellulose, lignin)

2ADF $=$ Acid Detergent Fibers (cellulose, lignin)

Table 3. Fiber analysis and process condition of feedstock and tested sample

\begin{tabular}{ccccccc}
\hline Feedstocks & Reaction & Residence & Heating rate & Cellulos & Hemicellulose & Lignin \\
& Temperature & time $(\mathrm{min})$ & $\left({ }^{\circ} \mathrm{C} \mathrm{min}^{-1}\right)$ & $\mathrm{e}(\%)$ & $(\%)$ & $(\%)$ \\
& $\left({ }^{\mathrm{o}} \mathrm{C}\right)$ & & & & & \\
\hline $\mathrm{CC}$ & - & - & - & 37.15 & 40.73 & 7.22 \\
$\mathrm{~B}-480$ & 480 & 15 & 10 & N/A & 2.69 & 89.36 \\
$\mathrm{H}-250$ & 250 & 100 & 5 & 46.12 & 35.23 & 10.26 \\
\hline
\end{tabular}

Table 4. BET surface area, BET C-constant and fiber analysis

\begin{tabular}{cccccc}
\hline Sample & BET surface area & BET & Cellulose & Hemicellulose & Lignin \\
& $\left(\mathrm{m}^{2} \mathrm{~g}^{-1}\right)$ & C-constant & $(\%)$ & $(\%)$ & $(\%)$ \\
\hline CC & 16.13 & 2.49 & 37.15 & 40.73 & 7.22 \\
B-480 & 7.66 & 6.46 & N/A & 2.69 & 89.36 \\
H-250 & 11.53 & 3.98 & 46.12 & 35.23 & 10.26 \\
\hline
\end{tabular}

\section{C.FT-IR Characteristics}

Carbon-oxygen surface groups are divided into 3 types as acidic, basic and neutral. In case of acidic surface groups, these render the carbon surface hydrophilic and polar in character and have been postulated to be carboxylic, lactone and phenolic groups. Pure carbon is hydrophobic in character. The amount of oxygen associated with the carbon surface increases in correspondence with the increasing of hydrophilic behavior. The presence of acidic

carbon-oxygen surface groups of active carbons makes the carbon surface hydrophilic and polar in character [10]. Fourier Transform Infrared (FT-IR) spectroscopy is the qualitative analysis aiming to find the functional group or oxygen surface group of substance. This result plots between wavenumber (cm-1) and \% Transmittance. FT-IR spectra of three sample (CC, H-250, B-480) shown in Figure 4.

Band of $\mathrm{CC}$ and $\mathrm{H}-250$ from this study are quite similar but slightly differ in intensity. B-480 quite flat in range
3500-1500 cm-1 and 1300-500 cm-1 differ vividly which represents the aromatic structure according to fiber analysis with $89.36 \%$ lignin. Oxygenated functional group

$(\mathrm{OFG})$ is represented in following bands : $3200 \mathrm{~cm}-1(\mathrm{O}$. $\mathrm{H}$ stretching, Hydroxyl or carbonyl groups (acid), alcohols from cellulose or phenols from lignin, $1725 \mathrm{~cm}-1(\mathrm{C} \cdot \mathrm{O}$ stretching, vibration in hemicellulose) which appear in $\mathrm{CC}$, decrease intensity $\mathrm{H}-250$ and rare in B-480 corresponding to BET surface area, $1240 \mathrm{~cm}-1$ (C • O stretching, hemicellulose esters), both $1205 \mathrm{~cm}-1$ (C $\cdot \mathrm{O}$ vibration in lignin) and $1149 \mathrm{~cm}-1$ (C $\cdot$ O stretching, aromatic skeletal) peak distinct in B-480 with higher lignin content and polymerize new aromatic skeletal, both 1020 and $1031 \mathrm{~cm}-1$ 


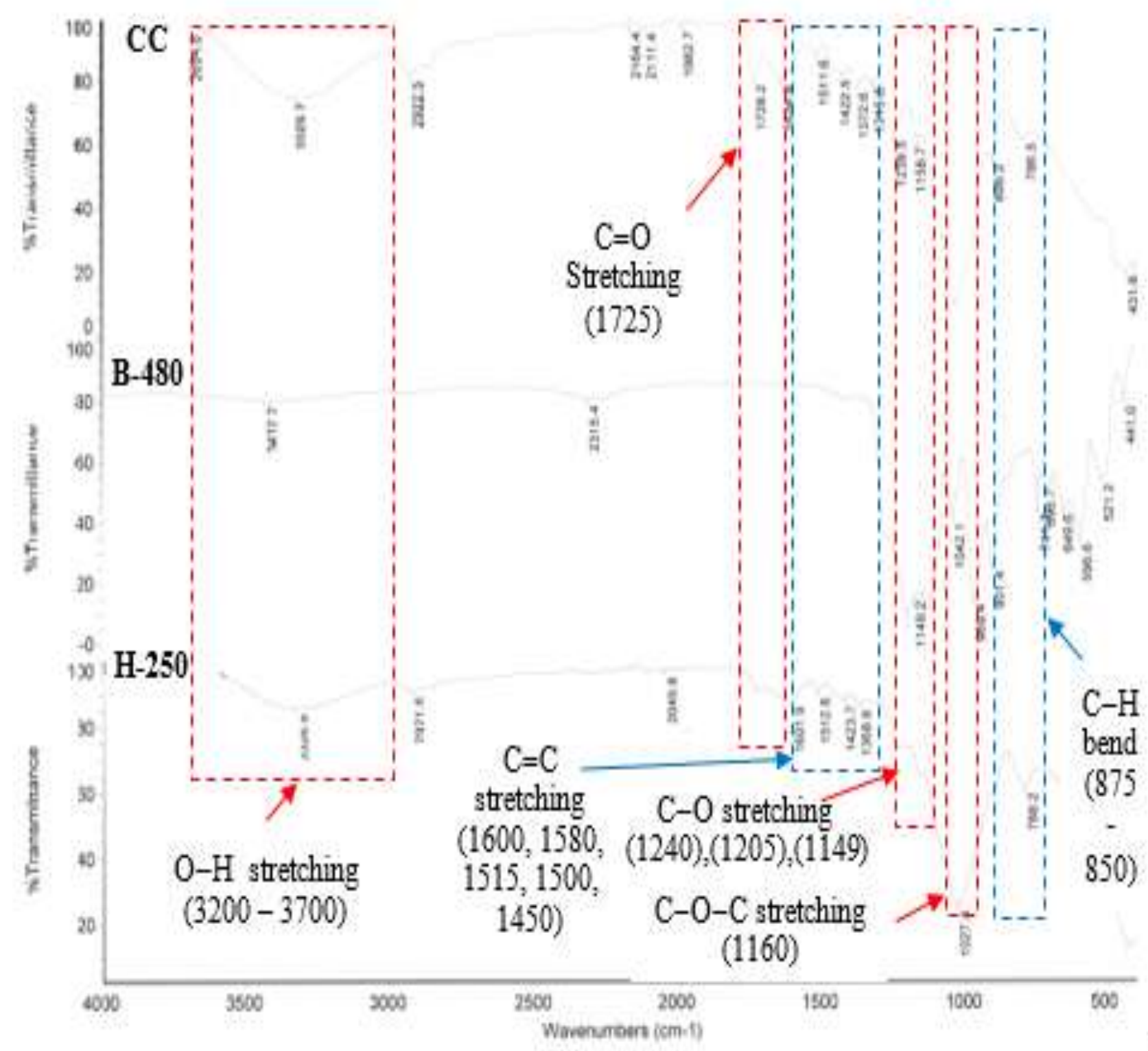

Figure 4. FT-IR spectra of Corncob feedstock (CC), Biochar produced at 480 o C (B-480) and Hydrochar produced at 250 o $\mathrm{C}(\mathbf{H}-250)$

(C - O stretching, vibrations in cellulose and hemicellulose) peak of $\mathrm{CC}$ and $\mathrm{H}-250$ more differ from B-480. The 1600, 1580, 1515, 1500 and $1450 \mathrm{~cm}-1(\mathrm{C} \cdot \mathrm{C}$ stretching, aromatic skeletal in lignin) indicate aromatic component in $\mathrm{CC}$ and $\mathrm{H}-250$ which are quite flat pattern in B-480. Their functional groups and description are described in Table 5.

\section{D.Discussion}

The three characterizations of corncob feedstock (CC) and carbonized corncob (B-480, H-250) include Fiber analysis (cellulose, hemicellulose and lignin constituents), FT-IR characteristic and BET surface area. The biochar from high temperature pyrolysis technique, B-480 char has high lignin content in which cellulose and hemicellulose are almost decomposed demonstrating the fully carbonized corncob with the lowest specific surface area of $7.66 \mathrm{~m} 2 \mathrm{~g}-1$. The carbonized corncob from mild temperature hydrothermal is partially carbonized with the $11.53 \mathrm{~m} 2 \mathrm{~g}-1$ BET surface area value. At this hydrothermal condition indicated that hemicellulose is destroyed partly. Therefore, this point is critical for optimization. The FT-IR result of $\mathrm{H}-250$ is distinct in oxygenated functional group differ from B-480 char. The amount of oxygen associated with the carbon surface of material increases in correspondence with the increasing of hydrophilic behavior. The aromatic structure of B-480 demonstrated the portion from the original feedstock and polymerized new aromatic skeletal. In addition, the aromatic portion in $\mathrm{B}-840$ is more distinct in $\mathrm{C} \cdot \mathrm{H}$ bend functional group comparing to $\mathrm{H}-250$. Therefore, the required and key elements characteristics of the carbonized corncob for further activation are high BET surface area, minimum hemicellulose constituent and more amount of oxygen on surface of bio-based carbon material via process optimization before activation step. 
International Conference on Recents Advancements in Engineering and Technology (ICRAET-18) |15th and 16th March 2019|Siddhartha Institute of Technology \& Sciences, Telangana, India

Table 5. Functional groups of feedstock and char derived from hydrothermal and pyrolysis [Modified from [24]]

\begin{tabular}{|c|c|c|}
\hline $\mathrm{cm}^{-1}$ & Functional group & Description \\
\hline $3200-3700$ & O-H stretching & $\begin{array}{l}\text { Hydroxyl or carbonyl groups (acid), alcohols from } \\
\text { cellulose or phenols from lignin (Strong and broad, quite } \\
\text { intense, smoothly curve) }\end{array}$ \\
\hline 2928,2870 & $\mathrm{C}-\mathrm{H}$ stretching & Aliphatic (Small double peak) \\
\hline 1725 & $\mathrm{C}=\mathrm{O}$ stretching & Vibration in hemicellulose \\
\hline 1700 & $\mathrm{C}=\mathrm{O}$ stretching & Carbonyl, ester or carboxyl from cellulose and lignin \\
\hline $1600,1580,1515,1500,1450$ & $\mathrm{C}=\mathrm{C}$ stretching & Aromatic skeletal in lignin \\
\hline 1240 & $\mathrm{C}-\mathrm{O}$ stretching & Hemicellulose esters \\
\hline 1205 & $\mathrm{C}-\mathrm{O}$ stretching & Vibrations in lignin \\
\hline 1149,1131 & $\mathrm{C}-\mathrm{O}$ stretching & Aromatic skeletal and stretching vibration in cellulose \\
\hline 1160 & $\mathrm{C}-\mathrm{O}-\mathrm{C}$ stretching & Vibrations in cellulose and hemicellulose \\
\hline 1103 & $\mathrm{C}-\mathrm{O}$ stretching & Vibrations in cellulose and lignin \\
\hline $1060,1020,1031$ & $\mathrm{C}-\mathrm{O}$ stretching & Vibrations in cellulose and hemicellulose \\
\hline $875-850$ & $\mathrm{C}-\mathrm{H}$ bend & Aromatic \\
\hline
\end{tabular}

also substantially involves the quantity of the oxygenated

\section{CONCLUSION}

This study aims to enhance the knowledge limitation regarding for key elements characteristics of carbonized corncob as the precursor material for further activation to produce the bio-based activated carbon via the mild temperature hydrothermal technique with demonstrated scale hydrothermal reactor comparing to high temperature pyrolysis. The fiber analysis, BET surface area and FT-IR analysis are key elements characteristics of both char from pyrolysis denoted as biochar and hydrothermal carbonization denoted as hydrochar. As for adsorption aspects, the adsorption capability is not only related to the surface area but functional surface group. The higher oxygenated functional groups contents and high BET surface area of hydrochar are the required characteristics of the precursor material for further activation which is predominant than biochar. However, the degradation of hemicellulose constituent is the crucial aspect.

Therefore, the future work will focus on the hydrothermal process optimization for better degradation of corncob constituents coupled with high oxygenated 
functional groups contents and high BET surface area as the precursor material for further activation to be utilized for bio-based activated carbon. Hydrothermal conversion also is environmental friendly technique with water as reaction medium. Feedstock biomass without drying step is also the advantage of hydrothermal process which differ from pyrolysis. It is important to note that the concept of conversion process and production conditions are important role in controlling char properties while feedstock constituent should be emphasized. Furthermore, the in-depth characterization clarify and enhance the correct upgrading requirement. A tremendous and exactly information of process conditions will scope quality, properties, direction of application because of changing in reaction resulted in kinetics and thermodynamics change. In addition, the hydrothermal process is the high pressure process in nature which the research gap regarding the safety aspect and simplified process still be possible.

\section{REFERENCES}

1. H. Chen, "Chemical Composition and Structure of Natural Lignocellulose," in Biotechnology of Lignocellulose : Theory and Practice: Dordrecht : Springer Netherlands : Springer, 2014, pp. 25-71.

2. E. Dinjus, A. Kruse, and N. Troger, "Hydrothermal Carbonization-1. Influence of Lignin in Lignocelluloses," (in English), Chemical Engineering \& Technology, Article vol. 34, no. 12, pp. 2037-2043, Dec 2011, doi: 10.1002/ceat.201100487.

A. Dimitriadis and S. Bezergianni, "Hydrothermal liquefaction of various biomass and waste feedstocks for biocrude production: A state of the art review," Renewable \& Sustainable Energy Reviews, vol. 68, pp. 113-125, Feb 2017, doi: 10.1016/j.rser.2016.09.120.

3. "Version 2.1 of the IBI Biochar Standards." (accessed 17-March 2018).

4. H. S. Kambo and A. Dutta, "A comparative review of biochar and hydrochar in terms of production, physico-chemical properties and applications," Renewable \& Sustainable Energy Reviews, vol. 45, pp. 359-378, May 2015, doi: 10.1016/j.rser.2015.01.050.

5. K. Qian, A. Kumar, H. Zhang, D. Bellmer, and R. Huhnke, "Recent advances in utilization of biochar," Renewable \& Sustainable Energy Reviews, vol. 42, pp. 1055-1064, Feb 2015, doi: 10.1016/j.rser.2014.10.074.

6. D. Mohan, A. Sarswat, Y. S. Ok, and C. U. Pittman, Jr., "Organic and inorganic contaminants removal from water with biochar, a renewable, low cost and sustainable adsorbent - A critical review," Bioresource Technology, vol. 160, pp. 191-202, May 2014, doi: 10.1016/j.biortech.2014.01.120.

7. G. K. Parshetti, S. K. Hoekman, and R. Balasubramanian, "Chemical, structural and combustion characteristics of carbonaceous products obtained by hydrothermal carbonization of palm empty fruit bunches," Bioresource Technology, vol. 135, pp. 683-689, May 2013, doi: 10.1016/j.biortech.2012.09.042.

8. Z. Liu, F.-S. Zhang, and J. Wu, "Characterization and application of chars produced from pinewood pyrolysis and hydrothermal treatment," Fuel, vol. 89, no. 2, pp. 510-514, Feb 2010, doi: 10.1016/j.fuel.2009.08.042.

9. L. Zhang, Q. Wang, B. Wang, G. Yang, L. A. Lucia, and J. Chen, "Hydrothermal Carbonization of Corncob Residues for Hydrochar Production," Energy \& Fuels, vol. 29, no. 2, pp. 872-876, Feb 2015, doi: 10.1021/ef502462p.

10. L. Zhang, S. Liu, B. Wang, Q. Wang, G. Yang, and J. Chen, "Effect of Residence Time on Hydrothermal Carbonization of Corn Cob Residual," Bioresources, vol. 10, no. 3, pp. 3979-3986, Aug 2015.

11. O. Arellano, M. Flores, J. Guerra, A. Hidalgo, D. Rojas, and A. Strubinger, "Hydrothermal Carbonization (htc) of Corncob and Characterization of the Obtained Hydrochar," Chemical Engineering Transactions, vol. 20, pp. 235-240, 2016, doi: 10.3303/CET1650040.

12. K. Nakason, B. Panyapinyopol, V. Kanokkantapong, N. Viriya-empikul, W. Kraithong, and P. Pavasant, "Characteristics of hydrochar and hydrothermal liquid products from hydrothermal carbonization of corncob," Biomass Conversion and Biorefinery, vol. 8, no. 1, pp. 199-210, Mar 2018, doi: 10.1007/s13399-017-0279-1.

13. M. Magdeldin, T. Kohl, and M. Jarvinen, "Techno-economic Assessment of Integrated Hydrothermal Liquefaction and Combined Heat and Power Production from Lignocellulose Residues," Journal of Sustainable Development of Energy Water and Environment Systems-Jsdewes, vol. 6, no. 1, pp. 89-113, Mar 2018, doi: 10.13044/j.sdewes.d5.0177.

14. R. C. Bansal and M. Goyal, Activated carbon adsorption. Boca Raton: Taylor \& Francis (in English), 2005.

15. Y. Chen et al., "Application studies of activated carbon derived from rice husks produced by chemical-thermal process-A review," Advances in Colloid and Interface Science, vol. 163, no. 1, pp. 39-52, Mar 15 2011, doi: 10.1016/j.cis.2011.01.006.

16. J. Chen, L. Zhang, G. Yang, Q. Wang, R. Li, and L. A. Lucia, "Preparation and Characterization of Activated Carbon from Hydrochar by Phosphoric Acid Activation and its Adsorption Performance in Prehydrolysis Liquor," Bioresources, vol. 12, no. 3, pp. 5928-5941, 2017 2017, doi: 10.15376/biores.12.3.5928-5941.

A. Kumar, L. Wang, Y. A. Dzenis, D. D. Jones, and M. A. Hanna, "Thermogravimetric characterization of corn stover as gasification and pyrolysis feedstock," Biomass \& Bioenergy, vol. 32 , no. 5, pp. 460-467, May 2008, doi: 10.1016/j.biombioe.2007.11.004.

17. A. Peterson, F. Vogel, R. P. Lachance, M. Froeling, M. J. Antal, Jr., and J. W. Tester, "Thermochemical biofuel production in hydrothermal media: A review of sub- and supercritical water technologies," Energy \& Environmental Science, vol. 1, no. 1, pp. 32-65, 2008 2008, doi: 10.1039/b810100k.

18. Funke and F. Ziegler, "Hydrothermal carbonization of biomass: A summary and discussion of chemical mechanisms for process engineering," Biofuels Bioproducts \& Biorefining-Biofpr, vol. 4, no. 2, pp. 160-177, Mar-Apr 2010, doi: 10.1002/bbb.198.

19. T. Wang, Y. Zhai, Y. Zhu, C. Li, and G. Zeng, "A review of the hydrothermal carbonization of biomass waste for hydrochar formation: Process conditions, fundamentals, and physicochemical properties," Renewable and Sustainable Energy Reviews, vol. 90, pp. 223-247, 2018/07/01/ 2018, doi: https://doi.org/10.1016/j.rser.2018.03.071

20. J. Mumme, L. Eckervogt, J. Pielert, M. Diakite, F. Rupp, and J. Kern, "Hydrothermal carbonization of anaerobically digested maize silage," Bioresource Technology, vol. 102, no. 19, pp. 9255-9260, Oct 2011, doi: 10.1016/j.biortech.2011.06.099. 
21. S. P. Sohi, E. Krull, E. Lopez-Capel, and R. Bol, "A REVIEW OF BIOCHAR AND ITS USE AND FUNCTION IN SOIL," Advances in Agronomy, Vol 105, vol. 105, pp. 47-82, 2010 2010, doi: 10.1016/s0065-2113(10)05002-9.

22. S. Guo, X. Dong, T. Wu, F. Shi, and C. Zhu, "Characteristic evolution of hydrochar from hydrothermal carbonization of corn stalk," Journal of Analytical and Applied Pyrolysis, vol. 116, pp. 1-9, Nov 2015, doi: 10.1016/j.jaap.2015.10.015. 